\title{
Tratamento cirúrgico das valvopatias. Parte 3
}

\author{
Domingo M. BRAILE* ${ }^{\star}$ Marco A. VOLPE**, Serginando L. RAMIN***, Dorotéia R. S. SOUZA****
}

RBCCV 44205-247

BRAILE, D. M.; VOLPE, M. A.; RAMIN, S. L.; SOUZA, D. R. S. - Tratamento cirúrgico das valvopatias. Parte 3. Rev. Bras. Cir. Cardiovasc., 9 (4): 181-192, 1994.

RESUMO: Este trabalho, subdividido em três partes, apresentou breve histórico da cirurgia cardiaca, com ênfase a cirurgia valvar e substitutos valvulares, empregados com sucesso na década de 60 , inicialmente com próteses mecânicas, seguidas pelas heterólogas após a introdução do glutaraldeído para preservação dos tecidos biológicos. As indicaçōes básicas para operar lesões valvares consistem em alívio dos sintomas, prevenção das complicaçōes e da mortalidade. Foram descritos, também, na primeira parte da publicação, aspectos da indicação cirúrgica, com ênfase em estenose e insuficiência das valvas mitral, aórtica, tricúspide e pulmonar, endocardite infecciosa ativa e da conduta pré-operatória, além da caracterização das diferentes próteses valvulares cardíacas mecânicas e biológicas existentes no mercado e suas complicaçōes mais frequientes. O tratamento cirúrgico das valvopatias, incluindo técnica operatória para troca de valvas mitral, aórtica, tricúspide e pulmonar, condutas anestésica e pós-operatória e reoperações foram abordados na segunda parte da publicação. O trabalho foi concluído considerando as situaçōes especiais, como tratamento cirúrgico na endocardite em valvas mitral, tricúspide e aórtica, cuja incidência é maior que na mitral e a causa mais comum de insuficiência aórtica aguda. O desenvolvimento da endocardite tem fisiopatologia diferente quando comparado às próteses e valvas naturais, com morbi-mortalidade maior que a observada nas valvas nativas. Existem fatores que aumentam o risco de endocardite em valva nativa, raça negra, próteses mecânicas, sexo masculino e longo tempo de circulação extracorpórea. A interação clínico-cirúrgica parece influenciar de forma decisiva na obtenção de melhores resultados para essa lesão. Finalmente, foi registrada a nossa experiência com próteses biológicas em posição mitral e aórtica com 11 e 10 anos de seguimento, respectivamente. $O$ indice de sobrevida na troca mitral foi semelhante entre jovens e adultos $(74 \%)$ para mitral e $67 \%$ para aórtica. As complicações tardias fatais relacionadas à bioprótese na posição mitral foram rotura, endocardite, vazamento paravalvular, tromboembolismo e, principalmente, calcificação, com freqũência de 1,0 evento \%/paciente-ano, com $95 \%$ dos pacientes livres dessas complicaçōes. Na posição aórtica, destacaram-se tromboembolismo e, principalmente, endocardite, totalizando 1,6 eventos \%/paciente-ano, com $92,6 \%$ dos pacientes livres dessas complicações. As complicações tardias não fatais apresentaram-se com freqüências de 2,9 (mitral) e 1,1 eventos \%/paciente-ano (aórtica), com destaque para endocardite e calcificação para mitral e endocardite e acidente vascular cerebral para aórtica, com $55,2 \%$ (mitral) e 85,7\% (aórtica) dos pacientes livres dessas complicações. Pela alta incidência de calcificação, principalmente entre pacientes jovens submetidos ao implante de biopróteses em geral, realizou-se um estudo abordando diferentes faixas etárias, com próteses de pericárdio bovino em posição mitral. Os resultados justificam ouso dessa bioprótese mesmo em pacientes jovens, contrariando autores que nāo recomendam o uso desse tipo de enxerto em pacientes com até 30 anos. Concluiu-se que a prótese de pericárdio bovino, quando rigorosamente preparada, apresenta, além de bom desempenho hemodinâmico, evolução tardia satisfatória. É importante considerar que a reunião permanente da literatura é fundamental para que a comunidade mantenha-se informada das vantagens e desvantagens de cada modelo de prótese disponível.

DESCRITORES: valvas cardiacas, cirurgia; valvopatias, história; valvopatias, indicação cirúrgica; valvopatias, conduta clínica; próteses valvulares cardiacas.

\footnotetext{
* Da UNICAMP/Campinas e FUNFARMES/S. J. Rio Preto, SP, Brasil.

** Do Hospital das Clínicas da UNICAMP. Campinas, SP.

*** De Braile Cardiocirurgia. São José do Rio Preto, SP.

**** De Braile Biomédica. São José do Rio Preto, SP.

Endereço para correspondência: Domingo Braile. Av. Juscelino Kubtschek, 3101. CEP 15091-450 - São José do Rio Preto, SP, Brasil. Tel. (0172)

274988 Fax: (0172) 273177.
} 
BRAILE, D. M.; VOLPE, M. A.; RAMIN, S. L.; SOUZA, D. R. S. - Tratamento cirúrgico das valvopatias. Parte $3 . \quad$ Rev. Bras. Cir. Cardiovasc., 9 (4): 181-192, 1994.

\section{SITUAÇÕES ESPECIAIS}

\section{Tratamento Cirúrgico na Endocardite}

A cirurgia tem um papel definido no tratamento da endocardite infecciosa, quer como método de escolha, quer como complemento à terapêutica medicamentosa.

$\mathrm{O}$ insucesso da antibioticoterapia como método isolado ocorre quando: 1) os agentes infecciosos são inacessíveis aos mecanismos de defesa do organismo (abscessos, grandes vegetações e pericardite purulenta); 2) há grande virulência do microorganismo causador, principalmente bactérias (Staphylococcus e gram negativos); 3 ) a terapêutica farmacológica não apresenta grande eficácia (endocardite fúngica) 12 .

A grande maioria dos pacientes, que requer cirurgia por endocardite na valva nativa, já apresentava uma valvopatia pregressa, congênita ou adquirida.

As cúspides valvares podem mostrar perfurações, fibroses e calcificações. Roturas de cordoalhas tendíneas ou de músculos papilares também são encontradas.

De maneira geral, a possibilidade de reparo cirúrgico está diretamente relacionada à extensão da destruição do anel e do grau de comprometimento das estruturas adjacentes ao mesmo. Os principios cirúrgicos na endocardite infecciosa consistem no desbridamento e remoção completa dos tecidos acometidos pelo processo infeccioso.

Uma das preocupações iniciais da realização de cirurgias cardíacas durante a endocardite ativa é o risco de infecção na prótese valvular, com índice de recorrência de $6 \% 32,36$.

É de grande importância a realização de imprint corado pelo método de Gram e de cultura do material obtido em campo operatório. Isto fornece excelente orientação para a antibioticoterapia. Preconiza-se que no campo operatório sejam usados antibióticos como gentamicina e/ou rifamicina no anel da prótese valvular antes de sua colocação no anel valvar nativo. Nos casos de endocardite fúngica, a irrigação do local com anfotericina $B$ parece ser eficaz. Acredita-se que a limpeza local com soluções anti-sépticas como povidine tópica possa ser benéfica. Outro auxiliar para este fim é a cola biológica, que parece ter importante efeito no combate à recidiva infecciosa.

Os antibióticos sistêmicos devem ser mantidos no pós-operatório por um período mínimo de 4 a 6 semanas.

\section{Endocardite valvar mitral}

A endocardite valvar mitral é menos freqüente quando comparada à da valva aórtica ${ }^{28}$.

Procedimentos de reparo podem ser feitos na valva mitral, incluindo o fechamento de pequenas perfurações em suas cúspides com auxílio de pericárdio bovino e a retirada de fragmentos de vegetações de suas cordas. Diante de uma leve disfunção causada por pequenas lesões, pode-se optar pela manutenção da valva adotando técnicas para sua reconstrução.

$\mathrm{Na}$ presença de grave comprometimento, é indicada a troca valvar ${ }^{14}$. Uma vez optado pela troca, deve ser feita uma rigorosa inspeção do anel valvar na busca de possíveis abscessos. É importante considerar também a possibilidade de endocardite valvar aórtica associada, não dispensando a inspeção deste aparelho valvar ${ }^{21}$.

Para a troca valvar mitral, procede-se conforme descrito anteriormente, exceto pelo fato da sutura ser feita com pontos de Poliéster ancorados em almofadas de Teflon ou pericárdio bovino.

A chave para a troca valvar na presença de endocardite é lembrar que os tecidos são muito friáveis e, para resultados hemodinâmicos satisfatórios a longo prazo, as suturas devem ser realizadas em tecido sadio e com pontos ancorados por almofadas de Teflon ou pericárdio bovino. Este tipo de sutura previne o vazamento paravalvular ${ }^{21}$.

$O$ anel mitral parece ser mais resistente à formação de abscessos do que o aórtico. $O$ tratamento cirúrgico da endocardite valvar mitral, com destruição do anel valvar, está associado com alta morbimortalidade. Isto deve-se, principalmente, às dificuldades em erradicar a infeç̧ão e também aos problemas técnicos para uma fixação adequada da prótese valvular em um anel fibroso comprometido ${ }^{15}$.

Pacientes com abscesso de anel representam um grande desafio ao cirurgião. A abordagem do abscesso deve ser feita por uma excisão de sua cavidade, desbridamento agressivo de todo o tecido necrótico, deixando os orifícios em comunicação com a corrente sangüínea sem obliterá-los com retalhos ou suturas 15,28 .

Até 0 início da década de 80 , o tratamento restringia-se ao desbridamento e fixação de uma prótese valvular em posição supra ou infra-anular no interior das cavidades atrial ou ventricular ${ }^{15}, 21$.

O tratamento cirúrgico dos abscessos anulares teve um grande avanço com a proposta de drenagem, seguida por reconstrução com pericárdio bovino ${ }^{15}$ (Figura 1). 
BRAILE, D. M.; VOLPE, M. A.; RAMIN, S. L.; SOUZA, D. R. S. - Tratamento cirúrgico das valvopatias. Parte $3 . \quad$ Rev. Bras. Cir. Cardiovasc., 9 (4): 181-192, 1994.

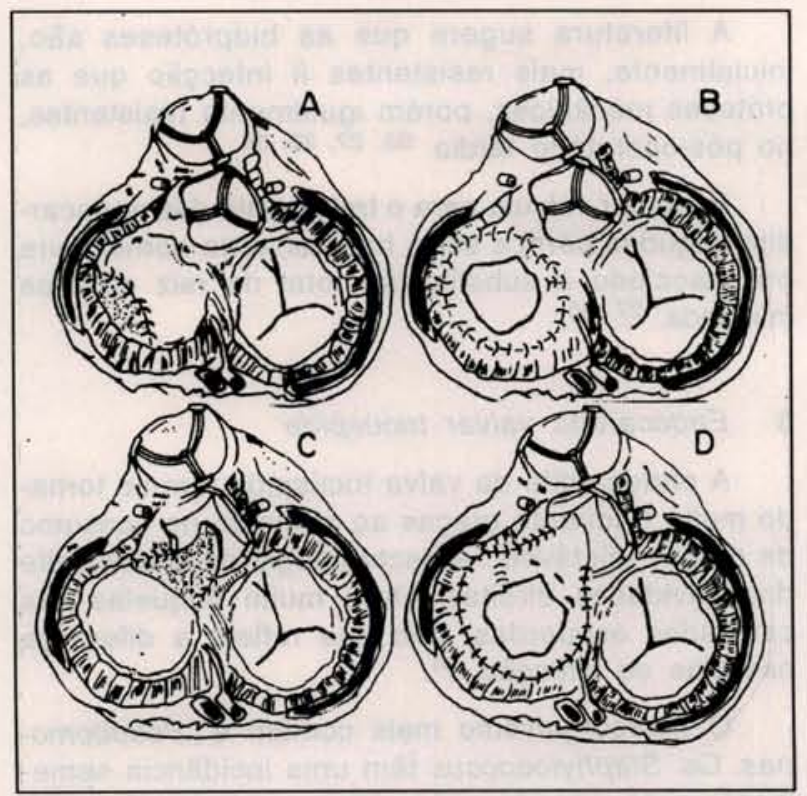

Fig. 1 - Reconstruçăo do anel mitral com retalho de pericárdio bovino. (A) Reconstrução de seguimento do anel posterior. (B) Reconstrução total do anel. (C) Reconstrução do trato de saida do ventrículo esquerdo. (D) Reconstrução total do ane e trato de saída do ventrículo esquerdo. David \& Feindel, $1987^{15}$.

O grau de comprometimento do anel tem níveis variáveis. Diante da destruição de pequenos segmentos do anel mitral posterior, preconiza-se a reconstrução com retalho semicircular de pericárdio bovino, suturado ao endocárdio do ventrículo esquerdo, contornando a cavidade do abscesso, iniciando e terminando no anel fibroso normal. A prótese mitral será suturada ao anel saudável e à borda livre do retalho de pericárdio bovino, suturado ao endocárdio do ventrículo esquerdo, contornando a cavidade do abscesso, iniciando e terminando no anel fibroso normal. A prótese mitral será suturada ao anel saudável e à borda livre do retalho de pericárdio (Figura 1A).

Nos casos de extensa destruição anular, uma reconstrução circunferencial com retalho de pericárdio bovino é indicada. Uma tira de pericárdio é suturada ao endocárdio do ventrículo esquerdo, posteriormente, e ao esqueleto fibroso, anteriormente. A prótese é fixada inteiramente neste novo anel de pericárdio (Figura 1B).

Quando o abscesso compromete o seio não coronariano da raiz aórtica e a cúspide anterior da valva mitral, pode-se proceder conforme descrito no tratamento cirúrgico da endocardite aórtica. Entretanto, existem situações em que o abscesso compromete o esqueleto fibroso central do coração. Nesses casos, os tratos de entrada e saída do ventrículo esquerdo tornam-se um orifício único. O esqueleto fibroso central pode ser substituído por um retalho triangular de pericárdio. A base deste triângulo servirá para a fixação de um segmento da prótese mitral, uma vez que o restante do anel mitral está íntegro. Os lados do triângulo serão suturados à parede ventricular e, posteriormente, à raiz da aorta (Figura $1 \mathrm{C}$ ).

Havendo comprometimento simultâneo do esqueleto fibroso central e de todo o anel mitral, o procedimento indicado é a reconstrução conjunta do anel e do esqueleto fibroso. O retalho de pericárdio com uma configuração adequada deverá ser suturado ao endocárdio do ventrículo esquerdo, como também à raiz da aorta, reconstruindo o anel mitral e parte do esqueleto fibroso central. Nesse caso, a prótese mitral será fixada inteiramente no retalho de pericárdio (Figura 1D).

O comprometimento simultâneo do anel mitral posterior e da parede posterior do ventrículo esquerdo pode ser tratado suturando-se um retalho semicircular de pericárdio bovino ao endocárdio do ventrículo esquerdo e terminando no remanescente do anel. A prótese valvular será fixada ao anel sadio e à borda livre do pericárdio ${ }^{15}$.

\section{Endocardite valvar aórtica}

A endocardite infecciosa é a causa mais comum de insuficiência aórtica aguda. Os agentes causais mais freqüentes são: cocos gram positivos (Staphylococcus e Streptococcus) e nos pacientes imunocomprometidos, os fungos. Os Streptococcus têm comportamento menos agressivo que os Staphylococcus, os quais, muito freqüentemente, acometem 0 anel, causando abscesso ${ }^{9}$.

As endocardites por Staphylococcus, por gram negativos e por fungos, mesmo sem sintomas, são indicações para cirurgia precoce. Mais raramente, o aparecimento de comunicações entre cavidades e os distúrbios de condução surgem como indicativos de cirurgia.

Quando a infecção acometer somente os folhetos valvares aórticos, os procedimentos de escolha são ressecção do aparelho valvar, e troca valvar conforme descrito anteriormente.

Nos casos em que o processo infeccioso afeta a raiz da aorta, são necessários procedimentos associados. Os fundamentos para o tratamento cirúrgico das infecções da raiz da aorta são: 1) erradicação da sepsis; 2) reparo dos defeitos associados relacionados à extensão da infecção; 3) uma firme fixação da válvula 9 .

Nos casos de abscessos sem grave destruição 
tecidual, a simples inclinação de uma válvula de baixo perfil, em relação ao anel, vai permitir a realização de uma sutura segura na parede aórtica não infectada.

Diante de uma destruição anular, adotamos a translocação do anel aórtico. Nesse caso, a válvula é transposta para uma posição supra-anular, sendo suturada na parede aórtica acima do anel. A esse procedimento deve associar-se a revascularização das artérias coronárias, já que a válvula encontrase em posição supracoronária $7,9,13,28$ (Figura 2).

A infecção da valva aórtica pode estar associada a aneurismas do seio de Valsalva. Se a abertura for pequena, pode ser fechada por sutura direta utilizando pontos com almofadas de Teflon ou pericárdio bovino. Nos casos de ampla abertura, geralmente o tecido é friável e aconselha-se a utilização de retalho de pericárdio para obliterar o aneurisma.

A extensão sub-anular da infecção é um problema particularmente difícil, que apresenta graus variáveis de acometimento das estruturas. Existem três grandes complicações da extensão sub-anular da infecção: 1) descontinuidade entre o ventrículo esquerdo e a aorta; 2) defeitos septais ventriculares; 3 ) infecção do anel mitral com descolamento da valva mitral. Estes defeitos merecem um tratamento especial, que deve ser particularizado de acordo com o tamanho dos mesmos, variando desde suturas com pontos ancorados em almofadas de Teflon ou pericárdio até a interposição de retalhos de pericárdio bovino ${ }^{9}$.

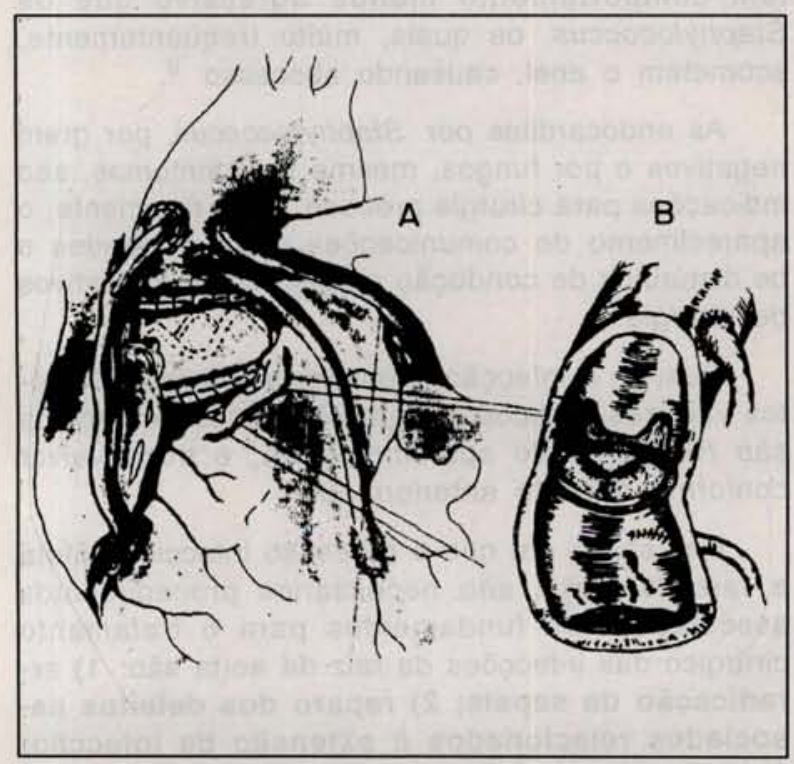

Fig. 2- Translocação do anel aórtico. (A) Prótese translocada superiormente. Adaptado de Khonsari, $1990^{22}$. (B) Visão com a aorta aberta. Adaptado de Mills, $1982^{28}$.
A literatura sugere que as biopróteses são, inicialmente, mais resistentes à infecção que as próteses mecânicas, porém igualmente resistentes, no pós-operatório tardio $23,27,30,35$.

A melhor válvula para o tratamento das endocardites agudas parece ser o homoenxerto aórtico livre ou associado à substituição total da raiz na área infectada 27,35 .

\section{Endocardite valvar tricúspide}

A endocardite da valva tricúspide tem-se tornado muito freqüente graças ao aumento no consumo de drogas injetáveis. A bacteriologia da endocardite das cavidades direitas difere muito daquelas das cavidades esquerdas, fato que reflete a diferença nas vias de infecção ${ }^{24}$.

O microorganismo mais comum é Pseudomonas. Os Staphylococcus têm uma incidência semelhante para as valvas de ambos os lados do coração. Não é incomum o acometimento da valva tricúspide por Candida, que é habitualmente de difícil tratamento 24 .

$\mathrm{Na}$ endocardite tricúspide pode-se ter acometimento do anel, das cúspides e/ou das cordoalhas. Os abscessos podem expor não apenas o anel, mas também o septo interventricular. As vegetaçōes chegam a apresentar tamanhos volumosos. Em consequeência do processo é possível ocorrer rotura de cordoalhas e, mesmo, destruição das cúspides.

O tratamento cirúrgico segue três diferentes caminhos: 1) Excisão, que consiste na exérese do aparelho valvar sem substituição por nenhuma prótese. Se necessário, pode ser feito um implante da mesma num segundo tempo. Esta proposta é particularmente útil nos casos em que o microorganismo causador tem grande virulência. 2) Substituição, nos casos em que há um importante comprometimento valvar e o microorganismo causador não é tão virulento. Neste caso adota-se a excisão seguida de implante de bioprótese. 3) Reparo valvar; faz-se a vegetectomia com desbridamento, provendo mais uma alternativa factível para o tratamento da endocardite tricúspide. Este é o método de escolha para os casos em que o microorganismo causador não é muito agressivo e o aparelho valvar não foi severamente lesado 12 .

Pacientes viciados em drogas injetáveis, que já tenham sido operados por endocardite, não têm indicação de reoperação por recidiva do processo infeccioso, caso não tenham deixado o vício.

\section{Endocardite em próteses valvulares}

O desenvolvimento da endocardite tem uma 
BRAILE, D. M.; VOLPE, M. A.; RAMIN, S. L.; SOUZA, D. R. S. - Tratamento cirúrgico das valvopatias. Parte $3 . \quad$ Rev. Bras. Cir. Cardiovasc., 9 (4): 181-192, 1994.

fisiopatologia diferente quando comparamos as próteses com as valvas naturais. A endocardite protética associa-se a uma morbi-mortalidade maior que a observada nas valvas nativas $2,14,34$. A literatura fornece índices de mortalidade que variam de $35 \%$ a $71 \%{ }^{18}$.

Os microorganismos mais comumente envolvidos são: Staphylococcus epidemides, Staphylococcus aureus, Streptococcus viridans e bactérias gram negativas 25 .

Um fator muito importante é o tipo de microorganismo que causa a endocardite na prótese. A mortalidade é mais alta nos casos em que temos germes invasivos e resistentes aos antibióticos.

O comprometimento dos tecidos adjacentes às válvulas, bem como do miocárdio, também são fatores que interferem aumentando a letalidade dessa lesão.

Genericamente as infecções em próteses cardíacas podem ser divididas em 21: a) Recentes, que aparecem antes de 60 dias de pós-operatório. Estas têm grande relação com contaminação per-operatória; são geralmente causadas por microorganismos mais virulentos (Staphylococcus, bactérias gram negativas e algumas vezes (Candida) e associam-se a alta mortalidade operatória. A virulência dos microorganismos envolvidos, a relação com contaminação per-operatória, bem como o acometimento do hospedeiro em período de convalescença e a queda na imunidade, causada pela CEC, favorecem o aparecimento de altas taxas de mortalidade relacionadas a infecção neste período. Uma vez tendo suportado uma nova cirurgia, estes pacientes têm uma sobrevida satisfatória. b) Tardias, que aparecem após 60 dias de pós-operatório. Estas relacionam-se com contaminação do meio ambiente e são geralmente causadas por microorganismos menos virulentos, tais como Streptococcus. Para estas infecções a sobrevida é comparável àquela da cirurgia para as endocardites de valva nativa, exceto a infecção por Staphylococcus.

Vários Serviços consideram a endocardite por Staphylococcus uma indicação formal para cirurgia, assim que firmado o diagnóstico. Devido a uma alta mortalidade notada retrospectivamente tanto para infecções recentes como tardias, e o conhecimento de grande incidência de destruição do anel valvar, sugerimos cirurgia para todos os casos de endocardite protética por Staphylococcus $14,20,21$.

As endocardites por fungos constituem por si uma indicação cirúrgica em qualquer momento, visto que não apresentam resposta satisfatória ao tratamento medicamentoso 20 .

A infecção em válvulas mecânicas geralmente acomete a sutura no anel, resultando em abscessos e graus variáveis de vazamento paravalvular 1, 14 . Não é incomum o acometimento de estruturas vizinhas, tais como abscessos miocárdicos, causando alterações de condução atrioventricular e fístulas para o pericárdio ou câmaras direitas. As vegetações são freqũentes e podem alcançar grandes tamanhos, comprometendo o funcionamento dos discos ou da bola nas próteses mecânicas ${ }^{21}$.

As infecções que acometem as biopróteses têm um espectro variável para o comprometimento da função valvular. As infecções recentes envolvem os folhetos ou o anel, ou ambos. As infecções tardias, via de regra, comprometem apenas os folhetos levando a um espessamento dos mesmos, fusão comissural e formação de grandes vegetações $14,29$.

Nas válvulas aórticas homólogas, a infecção inicia-se nos folhetos e os destrói rapidamente, mas raramente acomete 0 anel aórtico 8,14 .

Existem fatores que aumentam o risco de endocardite infecciosa em próteses valvulares, como: a) história de endocardite em valva nativa; b) raça negra; c) próteses mecânicas; d) sexo masculino; e) longo tempo de CEC ${ }^{21}$.

A cirurgia na endocardite protética deve ser indicada tendo como guia as alterações hemodinâmicas provocadas pela patologia e nunca o tempo de antibioticoterapia $12,14,32$. O princípio genérico que rege as cirurgias para endocardite protética é o da realização de amplo desbridamento cirúrgico, com remoção de todo o tecido perivalvular infectado. As técnicas para implante de nova prótese seguem aquelas descritas anteriormente. Devido à friabilidade dos tecidos, aconselha-se a realização de suturas com pontos envolvendo grande espessura de tecido sadio.

Os antibióticos específicos devem ser mantidos por um longo curso no pós-operatório após a retroca valvular.

O tratamento da endocardite protética pode ser tentado diante de: a) aparecimento tardio; b) micro-organismos não virulentos e sensíveis aos antibióticos; c) paciente hemodinamicamente compensado (ausência de insuficiência cardíaca congestiva e disfunção protética ausente ou mínima); d) ausência de eventos embólicos 18 .

O tratamento cirúrgico da endocardite protética continua sendo um tema muito controverso, em que cada grupo traça sua conduta baseando-se em resultados próprios. Existem grupos que preconizam o tratamento cirúrgico para todas as próteses mecânicas com endocardite, tão logo o diagnóstico tenha sido firmado ${ }^{14}$. Já outros tentam o tratamento medicamentoso, sempre que possível, e indicam 
BRAILE, D. M.; VOLPE, M. A.; RAMIN, S. L.; SOUZA, D. R. S. - Tratamento cirúrgico das valvopatias. Parte 3. Rev. Bras. Cir. Cardiovasc., 9 (4): 181-192, 1994.

tratamento cirúrgico, ulteriormente, caso este seja necessário 11.

A escolha da prótese na vigência de endocardite também é um tema discutível. Alguns sugerem que os bioenxertos teriam mais resistência à nova infecção $23,27,30,35$.

Insistimos no fato de que a nova cirurgia valvar deve ser indicada antes que as alterações hemodinâmicas tornem-se severas e irreversíveis, independente do tempo de antibioticoterapia. O adiamento da cirurgia, na tentativa de controlar a infecção, pode levar a um aumento do índice de morbidade e mortalidade per-operatória 32 .

A melhora dos resultados no tratamento cirúrgico da endocardite infecciosa deve-se a uma somatória de fatores: a) indicação cirúrgica em momento adequado; b) evolução nos métodos de proteção do miocárdio; c) aprimoramento das técnicas no manejo de pacientes com abscessos envolvendo extensa destruição do anel valvar e adjacências; d) aumento da experiência com reoperações valvulares; e) melhora nos métodos de controle dos distúrbios de coagulação; f) avanços tecnológicos no tratamento intensivo de pacientes com falência de múltiplos órgãos ${ }^{14}$.

Acreditamos que, em qualquer caso de endocardite protética, o paciente deva ser internado e o cirurgião, participado imediatamente na confirmação do diagnóstico. Uma interação clínico-cirúrgica parece influenciar de forma decisiva na obtenção de melhores resultados para esta lesão.

\section{EXPERIÊNCIA COM VÁLVULA DE PERICÁRDIO BOVINO}

Em nosso Serviço, acumulamos uma experiência de 20 anos com próteses biológicas, fato compreensível tendo em vista o baixo nível sócio-econômico e cultural da população a que se destina. A dificuldade do uso de anticoagulantes nesse grupo de pacientes justifica a preferência pelas próteses biológicas em detrimento das mecânicas, que ficam reservadas para casos muito selecionados.

Um seguimento de 11 anos com a prótese de pericárdio bovino implantada em 663 pacientes, com idade média de $40,0 \pm 14$ anos em posição mitral, revelou um desempenho satisfatório dessas biopróteses ${ }^{4}$. Nesse estudo, a mortalidade hospitalar foi de $9,2 \%$, principalmente por insuficiência ventricular esquerda. Para efeito comparativo os pacientes com óbito hospitalar foram divididos em dois grupos: Grupo I: composto por pacientes operados nos primeiros cinco anos, e Grupo II: pacientes operados nos últimos cinco anos. $O$ índice de mortalidade de $13,2 \%$ no primeiro período reduziu-se para $6,3 \%$ no segundo, sendo de $2,0 \%$ nos últimos dois anos, em face de $5,8 \%$ a $12 \%$ citados na literatura para esse mesmo tipo de bioprótese $3,5,6,33,37$. Dos pacientes que deixaram o hospital, $99,9 \%$ foram acompanhados, com seguimento médio de 3,8 anos e sobrevida de $74,3 \pm 6,5$ para os jovens e $73,0 \pm 3,7 \%$ para os adultos (Gráfico 1). Há referências de índices semelhantes $73,0 \pm 12,1 \% 37$ e inferior $54,0 \pm 6,0 \% 16$ em 10 anos de seguimento.

\section{GRÁFICO 1}

INDICES ATUARIAIS E LINEARIZADOS DE SOBREVIDA DE JOVENS E ADULTOS COM PRÓTESE DE PERICÁRDIO BOVINO EM POSIÇÃO MITRAL, COM 11 ANOS DE SEGUIMENTO

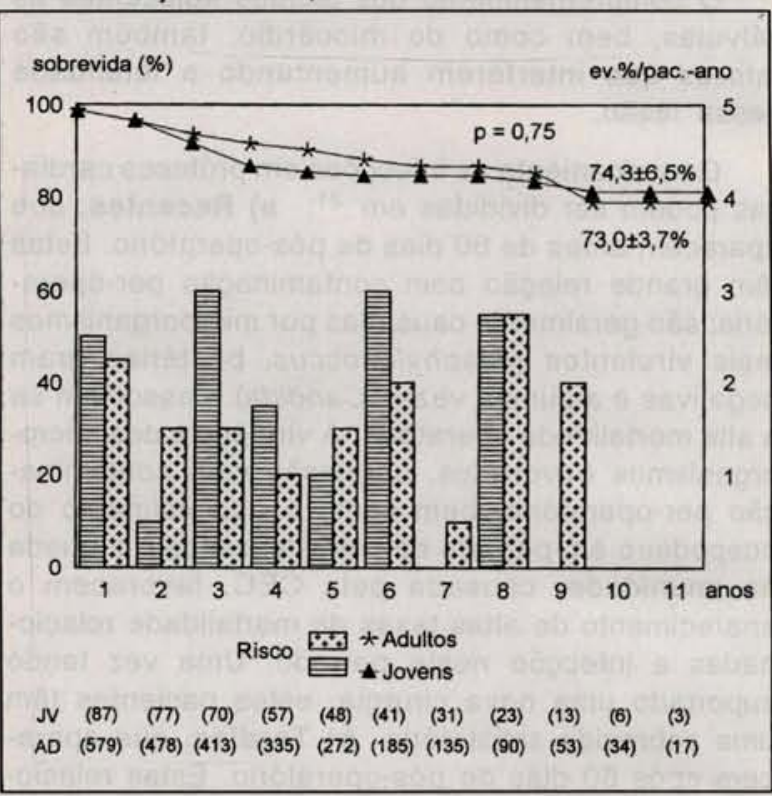

As complicações tardias fatais relacionadas à bioprótese, como calcificação, rotura, endocardite, vazamento paravalvular e tromboembolismo ocorreram com uma freqüência de 1,0 evento por cem paciente-ano, com $95,0 \pm 1,0 \%$ dos pacientes livres dessas complicações (Gráfico 2). Para as biopróteses em geral, a incidência linearizada global, nesse caso, foi de $5,2 \pm 0,5$ a $9,1 \pm 6,4$ eventos por cem paciente-ano 3,10 , em face do índice global de 3,9 eventos por cem paciente-ano, observados nesse estudo.

As complicações tardias não fatais foram detectadas com uma freqüência de 2,9 eventos por cem paciente-ano, com $55,2 \pm 8,6 \%$ dos pacientes livres dos eventos, sendo os mais freqüentes a endocardite $(0,5 \%$ /paciente-ano) e a calcificação $(1,8 \%$ /paciente-ano). Em estudo isolado de jovens ( $<=21$ anos) 
BRAILE, D. M.; VOLPE, M. A.; RAMIN, S. L.; SOUZA, D. R. S. - Tratamento cirúrgico das valvopatias. Parte $3 . \quad$ Rev. Bras. Cir. Cardiovasc., $9(4)$ : 181-192, 1994.

GRÁFICO 2

INDICES ATUARIAIS E LINEARIZADOS DE COMPLICAÇŌES FATAIS RELACIONADAS COM PRÓTESE DE PERICÁRDIO BOVINO EM POSICAAO AORTICA, COM 10 ANOS DE SEGUIMENTO

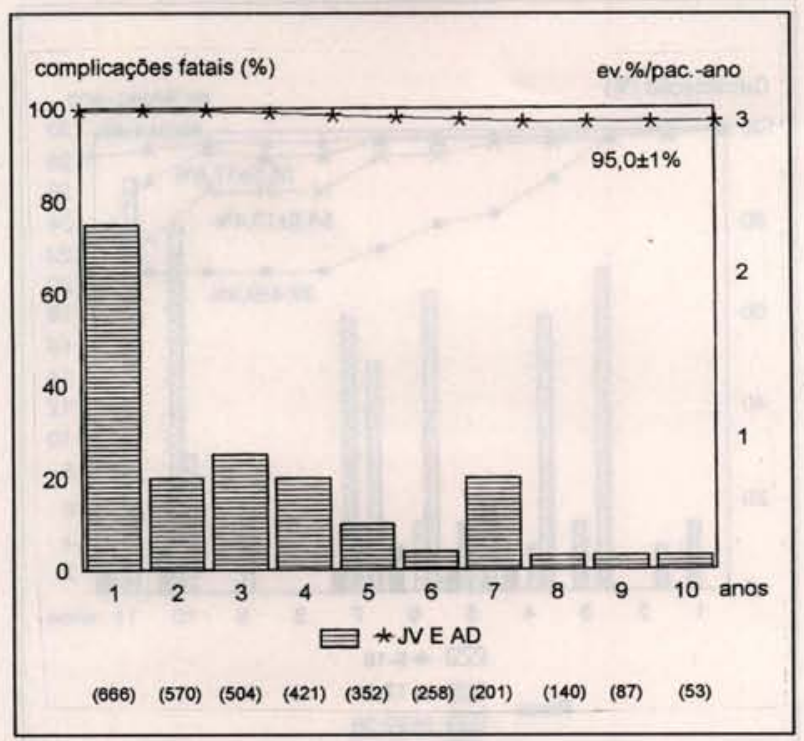

e adultos, registrou-se $35,6 \pm 13,4 \%$ dos jovens e $58,8 \pm 9,8 \%$ dos adultos livres de qualquer complicação, com incidência de 7,8 e 2,1 eventos por cem paciente-ano, respectivamente (Gráfico 3).

GRÁFICO 3

INDICES ATUARIAIS E LINEARIZADOS DE COMPLICAÇÕES NĀO FATAIS RELACIONADAS COM PRÓTESE DE PERICARDIO BOVINO EM POSIÇĀO MITRAL EM JOVENS E ADULTOS, COM 11 ANOS DE SEGUIMENTO

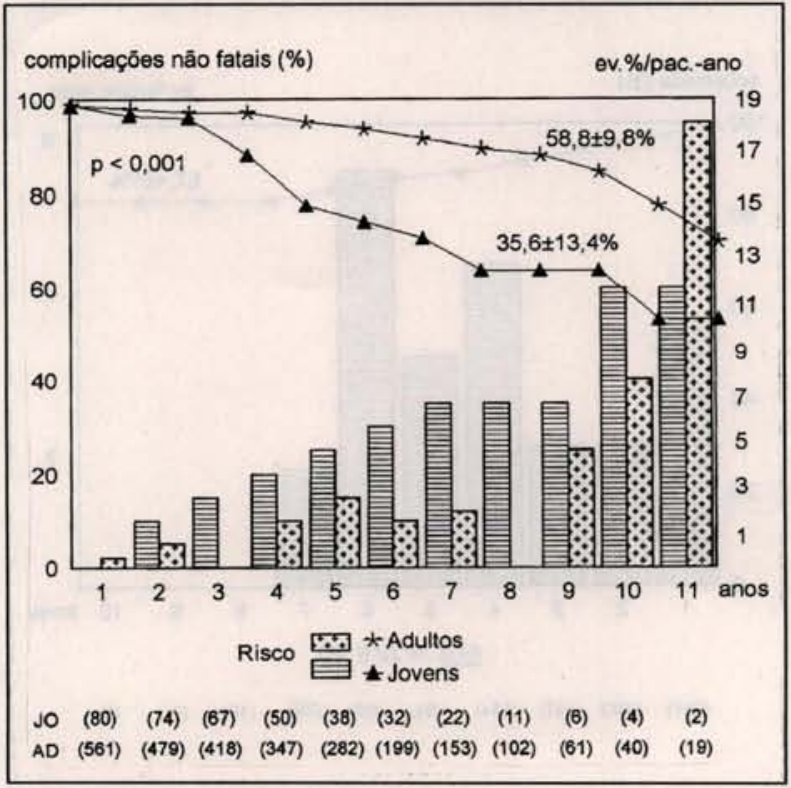

Considerando todas as complicações relacionadas a esse tipo de bioprótese, há referências de até 6,22 eventos por cem paciente-ano ${ }^{31}$.

O estudo atuarial revelou $95,8 \pm 1,6 \%$ de pacientes livres de tromboembolismo $(0,6$ eventos \%/paciente-ano). Os pacientes foram avaliados de acordo com a presença ou ausência de fibrilação atrial, no primeiro caso $92 \pm 2,9 \%$ ficaram livres de tromboembolismo; e entre aqueles com ritmo sinusal $98,0 \pm 1,1 \%$ ficaram livres desse evento (Gráfico 4 ).

Com relação às falhas primárias representadas por calcificação e rotura, $65,3 \pm 8,0 \%$ dos pacientes estiveram livres dessas complicaçōes, correspondendo a 1,9 e 0,08 eventos por cem paciente-ano, respectivamente. Em estudo isolado de jovens e adultos detectou-se $43,1 \pm 12,2 \%$ de jovens e $68,4 \pm 9,3 \%$ de adultos livres dessas complicações (Gráfico 5). Na literatura são encontrados para esse mesmo tipo de bioprótese índices de $70,0 \pm 11,0 \%$ $(1,77$ eventos por cem paciente-ano) 31 e $44,0 \pm$ $11,0 \%{ }^{26}$ de pacientes livres de falhas primárias em 6 a 10 anos de seguimento, respectivamente, em face dos índices de $88 \%$ e $78 \%$, encontrados nesse estudo para os mesmos seguimentos.

Pela importância da calcificação, principalmente para os pacientes jovens, foi realizado um estudo abordando diferentes faixas etárias, revelando que, entre os pacientes com 5 a 16 anos, $39,4 \pm 9,9 \%$

\section{GRÁFICO 4}

INDICES ATUARIAIS ELINEARIZADOS DE TROMBOEMBOLISMO EM PACIENTES COM PROTTESE DE PERICARDIO BOVINO EM POSIÇÃO MITRAL, COM 11 ANOS DE SEGUIMENTO

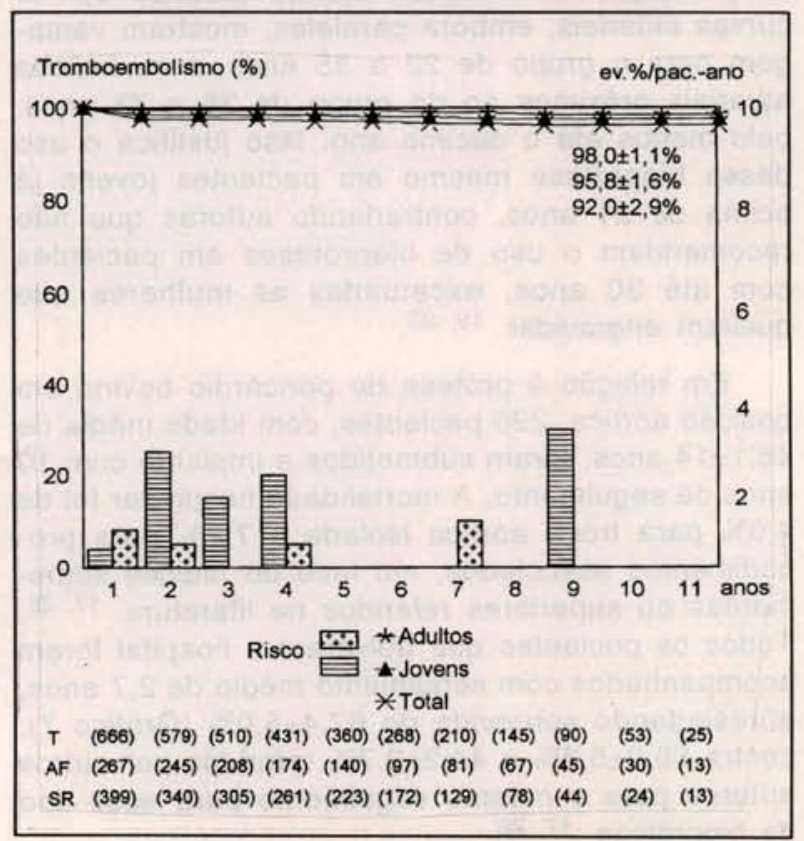


BRAILE, D. M.; VOLPE, M. A.; RAMIN, S. L.; SOUZA, D. R. S. - Tratamento cirúrgico das valvopatias. Parte $3 . \quad$ Rev. Bras. Cir. Cardiovasc., 9 (4): 181-192, 1994.

GRÁFICO 5

INDICES ATUARIAIS E LINEARIZADOS DE FALHAS PRIMÁRIAS EM JOVENS E ADULTOS COM PRÓTESE DE PERICÁRDIO BOVINO EM POSIÇĀO MITRAL, COM 11 ANOS DE SEGUIMENTO

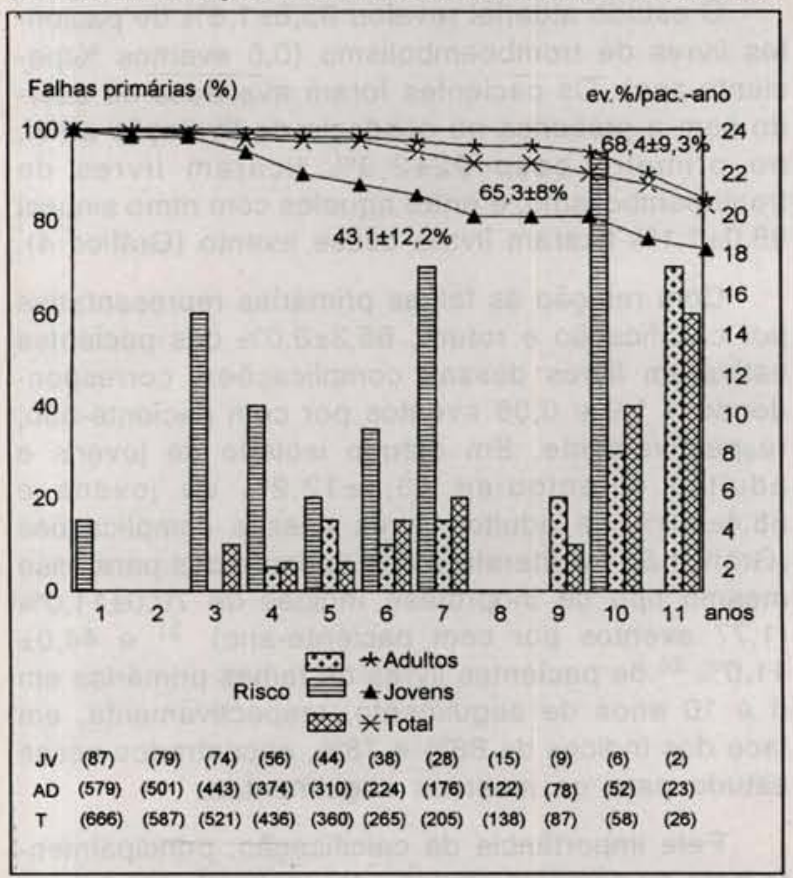

ficaram livres de calcificação (12,0\%/paciente-ano), para os de 17 a 21 anos e 22 a 35 anos os índices de pacientes livres dessa complicação foram $54,8 \pm 13,4 \%(3,8 \% /$ paciente-ano $)$ e $55,5 \pm 17,6 \%$ $(1,6 \% /$ paciente-ano), respectivamente, e entre aqueles de 36 a 73 anos, $89,2 \pm 4,8 \%(0,7 \% /$ pacienteano) ficaram livres desse evento (Gráfico 6). As curvas atuariais, embora paralelas, mostram vantagem para o grupo de 22 a 35 anos, com índices atuariais próximos ao do grupo de 36 a 73 anos, pelo menos até o décimo ano. Isso justifica o uso dessa bioprótese mesmo em pacientes jovens já acima de 21 anos, contrariando autores que não recomendam o uso de biopróteses em pacientes com até 30 anos, excetuadas as mulheres que queiram engravidar 19,33 .

Em relação à prótese de pericárdio bovino em posição aórtica, 296 pacientes, com idade média de $48,1 \pm 14$ anos, foram submetidos a implante com 10 anos de seguimento. A mortalidade hospitalar foi de $4,6 \%$ para troca aórtica isolada e $7,7 \%$ para procedimentos associados, em face de índices semeIhantes ou superiores referidos na literatura 17, 26 . Todos os pacientes que deixaram o hospital foram acompanhados com seguimento médio de 2,7 anos, apresentando sobrevida de $67,4 \pm 5,0 \%$ (Gráfico 7 ), contra $56,0 \pm 5,8 \%$ e $44,2 \pm 9,7 \%$ referidos por outros autores para o mesmo seguimento para esse tipo de bioprótese 17, 26
GRÁFICO 6

INDICES ATUARIAIS E LINEARIZADOS DE CALCIFICAÇÃO DA PRÓTESE DE PERICÁRDIO BOVINO EM POSIÇĀO MITRAL POR FAIXA ETÁRIA, COM 11 ANOS DE SEGUIMENTO

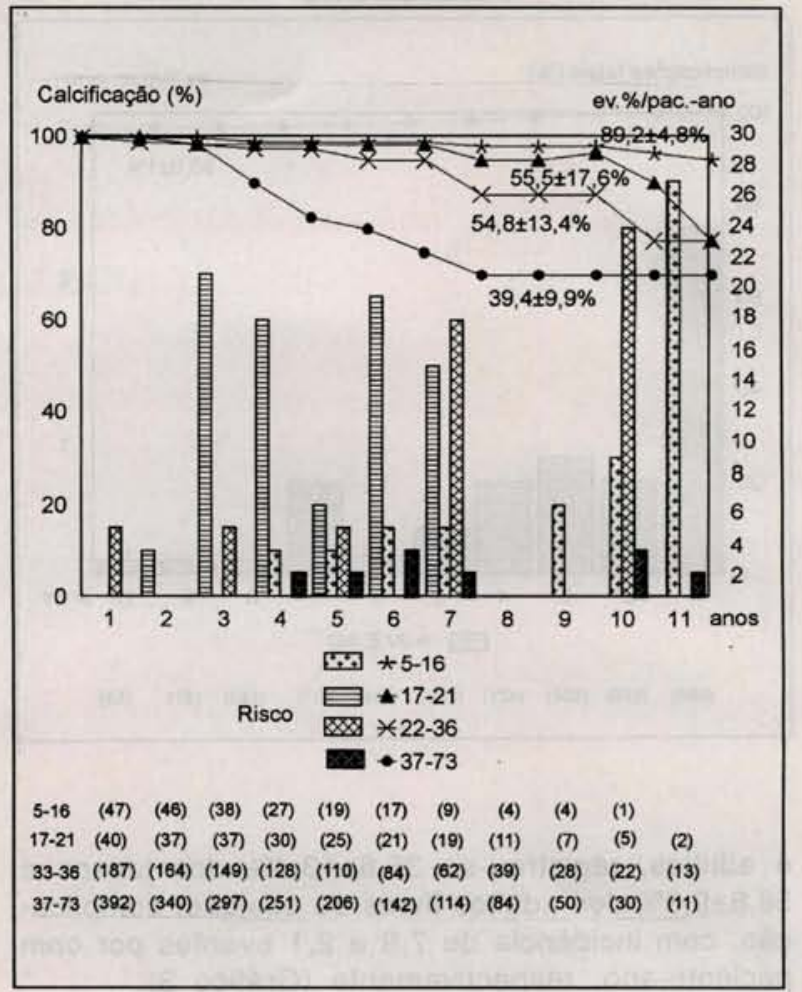

GRÁFICO 7

INDICES ATUARIAIS E LINEARIZADOS DE SOBREVIDA DE JOVENS E ADULTOS COM PRÓTESE DE PERICÁRDIO BOVINO EM POSIÇÃO AORTICA, COM 10 ANOS DE SEGUIMENTO

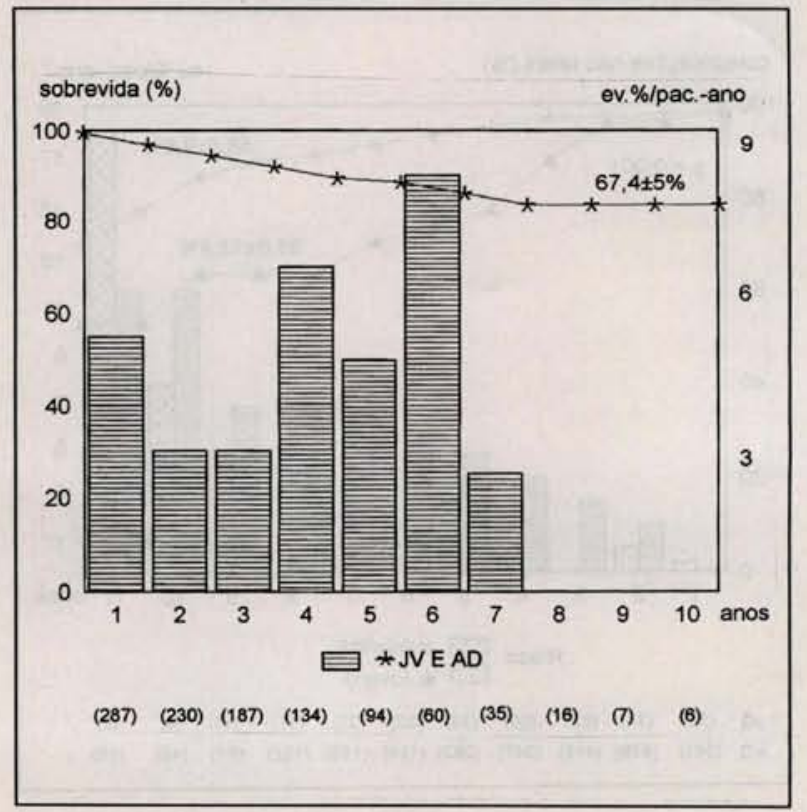


BRAILE, D. M.; VOLPE, M. A.; RAMIN, S. L.; SOUZA, D. R. S. - Tratamento cirúrgico das valvopatias. Parte $3 . \quad$ Rev. Bras. Cir. Cardiovasc., 9 (4): 181-192, 1994.

As complicações tardias fatais relacionadas à bioprótese foram, entre outras, tromboembolismo e principalmente endocardite, totalizando 1,6 eventos por cem paciente-ano, com $92,5 \pm 2,4 \%$ dos pacientes livres dessas complicações nesse período (Gráfico 8$)$, índice comparado a $92,2 \pm 3,0 \%(0,8$ eventos por cem paciente-ano), referido na literatura ${ }^{17}$. Entre as complicações tardias não fatais relacionadas à bioprótese destacaram-se a endocardite e o acidente vascular cerebral, seguidos de calcificação, rotura e vazamento paravalvular, totalizando 1,1 eventos por cem paciente-ano, com $85,7 \pm 4,2 \%$ dos pacientes livres dessas complicações (Gráfico 8), entretanto, é referido na literatura índice inferior $(40,9 \pm$ $10,4 \%)^{17}$. Para o tromboembolismo $96,7 \pm 1,8 \%$ dos pacientes ficaram livres desse evento sem o uso de anticoagulantes, em face de $89,9 \pm 3 \%$ registrado na literatura para seguimento semelhante 17 .

\section{GRÁFICO 8}

INDICES ATUARIAIS E LINEARIZADOS DE COMPLICAÇÕES FATAIS E NÃO FATAIS RELACIONADAS COM PRÓTESE DE PERICÁRDIO BOVINO EM POSIÇĀO AORTICA, COM 10 ANOS DE SEGUIMENTO

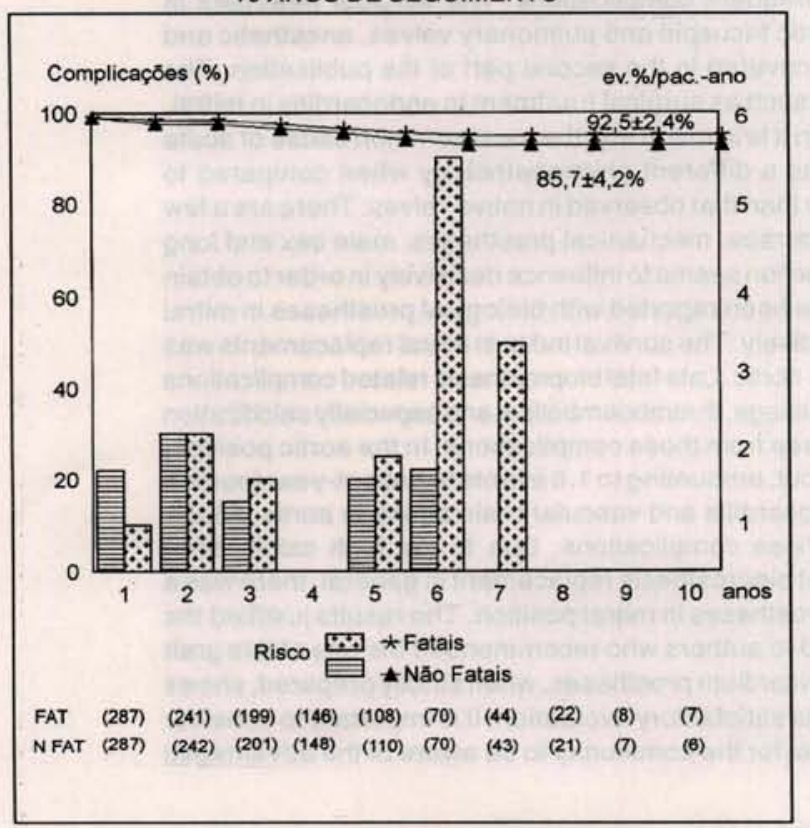

As falhas primárias representadas por calcificação e rotura corresponderam a 0,5 eventos por cem paciente-ano, com $100 \%$ dos jovens e $92,7 \pm$ $3,7 \%$ dos adultos livres dessas complicações (Gráfico 9). Um total de 2,4 eventos por cem paciente-

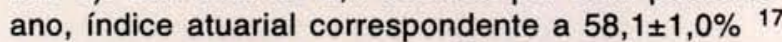
e até inferior de $48,0 \pm 7,0 \% 26$ de pacientes livres de falhas intrínsecas do tecido foram referidas em
GRÁFICO 9

INDICES ATUARIAIS E LINEARIZADOS DE FALHAS PRIMÁRIAS PARA JOVENS E ADULTOS COM PRÓTESE DE PERICÁRDIO BOVINO EM POSIÇÃO AORTICA, COM 10 ANOS DE SEGUIMENTO

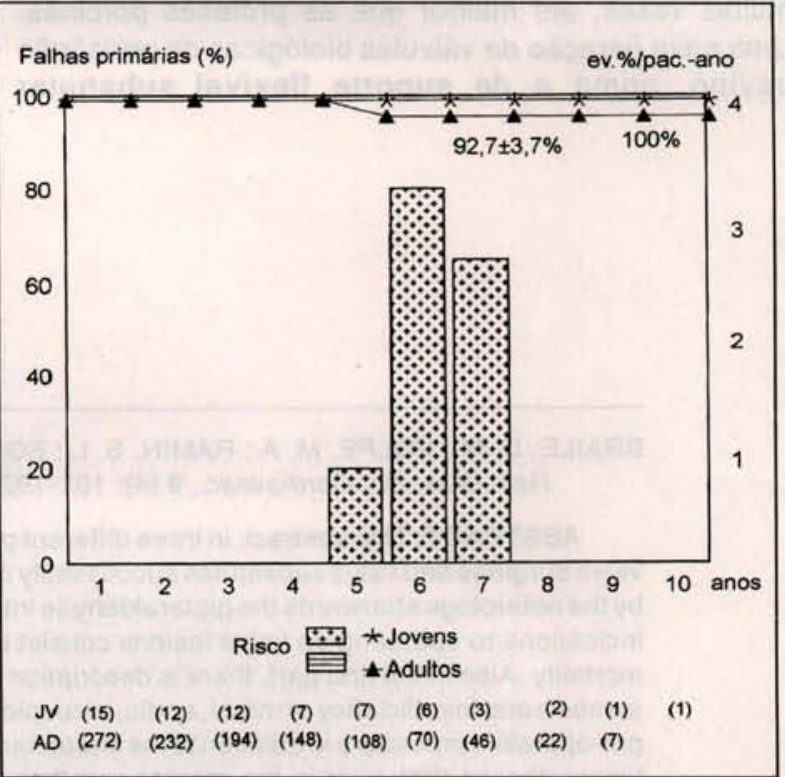

10 anos para esse mesmo tipo de bioprótese. A calcificação isolada foi detectada com incidência de $0,2 \% /$ paciente-ano, correspondendo a $100 \%$ dos jovens e $95,4 \pm 3,3 \%$ dos adultos livres desse evento (Gráfico 10).

\section{GRÁFICO 10}

INDICES ATUARIAIS E LINEARIZADOS DE CALCIFICACÃO EM JOVENS E ADULTOS COM PROTTESE DE PERICÁRDIO BOVINO EM POSICYAOO AÓRTICA, COM 10 ANOS DE SEGUIMENTO

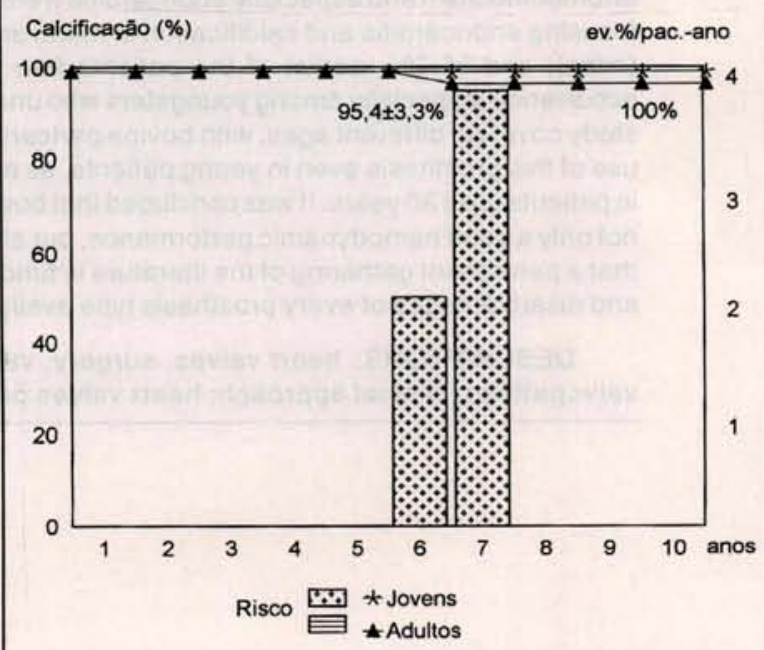

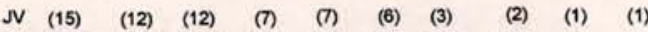

$\begin{array}{llllllllllll}A D & (272) & (232) & (194) & \text { (148) } & \text { (107) } & \text { (69) } & \text { (46) } & \text { (22) } & \text { (7) }\end{array}$ 
A experiência apresentada permite-nos concluir que a prótese de pericárdio bovino quando rigorosamente preparada apresenta além de bom desempenho hemodinâmico, evolução tardia comparável e, muitas vezes, até melhor que as próteses porcinas. Uma nova geração de válvulas biológicas de pericárdio bovino, como a de suporte flexível subanular
(Carpentier) e a integral (Braile), têm mostrado desempenhos competitivos, mesmo quando comparadas às próteses mecânicas modernas, podendo prescindir do uso de anticoagulantes. A revisão permanente da literatura é fundamental para que a comunidade fique sempre informada das vantagens e desvantagens de cada modelo de prótese disponível.

RBCCV 44205-247

BRAILE, D. M.; VOLPE, M. A.; RAMIN, S. L.; SOUZA, D. R. S. - Valvopathies: surgical treatment. Part 3. Rev. Bras. Cir. Cardiovasc., 9 (4): 181-192, 1994.

ABSTRACT: This abstract, in three different parts, has presented a briefing on cardiac surgery focusing valve surgiries and valve substitutes successfully used in the 60 's at first the mechanical prosthesis, followed by the heterologs afterwards the glutaraldehyde introduction in order to preserve biological tissues. The basic indications to operating on valve lesions consist in symptom relief in preservation of complications and in mortality. Also in the first part, there is description of the surgical indication publication aspects pointing out stenosis and insufficiency of mitral, aortic, tricuspid and pulmonary valves, active inffectious endocarditis and pre-operative procedure in addition to the characterization of different mechanical and biological cardiac valve bioprostheses that exist in the market and their most frequent complications. The surgical treatment in valvopathies, operative techniques to replace mitral, aortic tricuspid and pulmonary valves, anesthetic and post-operative procedure as well as reoperations were covered in the second part of the publication. The abstract was concluded considering particular situations such as surgical treatment in endocarditis in mitral, tricuspid and aortic valves whose tendency is greater than it is in mitral and the most common cause of acute aortic failure as well. The endocarditis development has a different physiopathology when compared to prostheses and native valves, with greater morbi-mortality than that observed in native valves. There are a few endocarditis increasing risk factors in native valves, black race, mechanical prostheses, male sex and long extracorporeal circulation time. The clinical-surgical interaction seems to influence decisively in order to obtain better results in this pathology. Finally our experience has been reported with biological prostheses in mitral and aortic positions in 11 and 10 years of follow-up, respectively. The survival index in mitral replacements was similar amongst youngsters and adults $74 \%$ mitral and $67 \%$ aortic. Late fatal bioprosthesis related complications in mitral position were rupture, endocarditis, paravalvular leakage, thromboembolism and especially calcification at a rate of 1.0 event $\%$ patient-year $95 \%$ of the patients free from those complications. In the aortic position, thrombombolism and especially endocarditis were ruled out, amounting to 1.6 events \% patient-year (aortic), focusing endocarditis and calcification in mitral and endocarditis and vascular brain stroke in aortic, $55.2 \%$ (mitral) and $85.7 \%$ (aortic) of the patients free from those complications. Due to the high calcification occurrence, especially among youngsters who underwent bioprosthesis replacement in general, there was a study covering different ages, with bovine pericardium prostheses in mitral position. The results justified the use of this prosthesis even in young patients, as apposed to authors who recommended the use of this graft in patients up to 30 years. It was concluded that bovine pericardium prostheses, when strictly prepared, shows not only a good hemodynamic performance, bur also a late satisfactory evoluation. It is important to consider that a permanent gathering of the literature is fundamental for the community to be aware of the advantages and disadvantages of every prosthesis type available.

DESCRIPTORS: heart valves, surgery; valvopathies, history; valvopathies, surgical indications; valvopathies, clinical approach; heart valves prostheses. 
BRAILE, D. M.; VOLPE, M. A.; RAMIN, S. L.; SOUZA, D. R. S. - Tratamento cirúrgico das valvopatias. Parte $3 . \quad$ Rev. Bras. Cir. Cardiovasc., 9 (4): 181-192, 1994.

\section{REFERÊNCIAS BIBLIOGRÁFICAS}

1 ARNETT, R. N. \& ROBERTS, W. C. - Prosthetic valve endocarditis: clinical pathologic analysis of 22 necropsy patients with comparison of observation in 74 necropsy patients with active infective endocarditis involving natural left-sided cardiac valves. Am. J. Cardiol., 38: 281-292, 1976.

2 BAumgartneR, W. A.; MILleR, D. C.; REITZ, B. A. - Surgical treatment of prosthetic valve endocarditis. Ann. Thorac. Surg., 35: 87-104, 1983.

3 BORTOLOTTI, U.; MILANO, A.; THIENE, G.; GUERRA, F.; MAZZUCCO, A.; VALENTE, M.; TALENTI, E.; GALLUCCI, V. - Early mechanical failures of the Hancock pericardial xenograft. J. Thorac. Cardiovasc. Surg., 94: 200-207, 1987.

4 BRAILE, D. M. - Prótese valvular de pericárdio bovino: desenvolvimento $\theta$ aplicação clínica em posição mitral, São Paulo, 1990. [Tese Doutorado]. Escola Paulista de Medicina. 110p.

5 BRAILE, D. M.; ZAIANTCHICK, M.; SOUZA, D. R. S. Prótese de pericárdio bovino PB IMC: 11 anos em posição mitral. Arq. Bras. Cardiol., 59: 13-21, 1992.

6 BRICK, A. V.; MIANA, A. A.; COLEN, E. A.; PASSOS, P. H. C.; BORGES, A.; JORGE, P. C.; BRAILE, D. M.; GRECO, O. T.; ARDITO, R. V.; SANTOS, J. L. V.; MAYORQUIM, R. C.; LIMA, E. R.; ZAIANTCHICK, M.; CAMPOS, N. L. K. L.; GOLLARZA, H. S.; SOUZA, D. R. S.; BROFMAN, P. R.; LOURES, D. R. R.; CARVALHO, R. G.; RIBEIRO, E. J. - Seguimento de 9 anos de bioprótese valvar cardiaca de pericárdio bovino IMC-Biomédica: estudo multicêntrico. Rev. Bras. Cir. Cardiovasc., 2: 189-190, 1987.

7 CABROL, C.; GANDJBAKHCH, I.; PAVIE, A.; BORS, V.; CABROL, A.; LEGER, P.; LEVASSEUR, J. P.; VAISSIER, E.; PETRIE, J. - Approach to advanced root infection. J. Cardiac. Surg., 5: 48-52, 1990.

8 CLARKSON, P. M. \& BARRATT-BOYES, B. G. Bacterial endocarditis following homograft replacement of the aortic valve. Circulation, 42: 987 $994,1970$.

$9 \mathrm{COHN}$, L. H. - Tratamento da insuficiência aórtica aguda. In: ARAÚJO, M. A. C. \& LANA, R. L., (trads.) Clínicas cardiológicas: doença cardiaca valvular. Rio de Janeiro, Interlivros, 1991. p. 363-377.

10 COHN, L. H.; ALLRED, E. N.; COHN, L. A.; AUSTIN, J. C.; SABIK, J.; DISESA, V. J.; SHEMIN, R. J.; COLLINS Jr., J. J. - Early and late risk of mitral valve replacement: a 12-year concomitant comparison of the porcine bioprosthetic and prosthetic disc mitral valves. J. Thorac. Cardiovasc. Surg., 90: 872-881, 1985.

11 COHN, L. H.; DOTY, D. B.; MCELVEIN, R. B. - Decision making in cardiothoracic surgery. Philadelphia, BC Decker, 1987, p. 14-15.
COUTO, A.; RANGEL, F.; CARNEIRO, R. - Indicaçōes cirúrgicas na endocardite infecciosa. In: COUTO, A. A. \& CARNEIRO, R. D., (eds.) Indicações cirúrgicas em cardiologia. Rio de Janeiro, Atheneu, 1991. p. 63 74.

13 DANIELSON, G. K.; TITUS, L. J.; DUSHANE, J. W. Surgical treatment of aortic root abscess by insertion of prosthetic valve in ascending aorta and placement of bypass grafts to coronary aorta arteries. $J$. Thorac. Cardiovasc. Surg., 67: 443-446, 1974.

DAVID, T. E.; BOS, J.; CHRISTAKIS, G. T.; BROFMAN, P. R.; WONG, D.; FEINDEL, C. M.- Heart valve operations in patients with active infective endocarditis. Ann. Thorac. Surg., 49: 701-705, 1990.
15

16

17
DAVID, T. E.; \& FEINDEL, C. M. - Reconstruction of mitral annulus. Circulation, 76: 102-107, 1987.

EDMUNDS Jr., L. H. - Thromboembolic complications of current cardiac valvular prostheses. Ann. Thorac. Surg., 34: 96-106, 1982.

GONZALEZ-LAVIN, L.; GONZALEZ-LAVIN, J.; CHI, S.; LEWIS, B.; AMINI, S.; GRAF, D. - The pericardial valve in the aortic position ten years later. J. Thorac. Cardiovasc. Surg., 101: 75-80, 1991.

ISMAIL, M. B.; ABID, F.; HANNACHI, N.; KAABAR, Z.; ROUGÉ, J. F. - Endocarditis following cardiac valve replacement. Cardiol. Board. Rev., 5: 115-125, 1988.

JAMIESON, W. R. E.; ROSADO, L. J.; MUNRO, A. I.; GEVERN, A. N.; BURR, L. H.; MIYAGISHIMA, R. T.; JANUSZ, M. T.; TYERS, G. F. - Carpentier-Edwards standard porcine bioprosthesis: primary tissue failure (structural valve detereoration) by age groups. Ann. Thorac. Surg., 46: 155-162, 1988.

KARCHMER, A. W. - Prosthetic valve endocarditis. In: FORTUIN, N. J. Current therapy in cardiovascular disease. Philadelphia, BC Decker, 1987. p. 155-158.

KARP, R. B. - Role of surgery in infective endocarditis. In: McGOON, D. C. Cardiac surgery. 2. ed. Philadelphia, FA Davis, 1987 p. 141-162.

KHONSARI, S. - Atlas de Cirurgia cardiaca: cuidados em técnica operatória. GELMAN, A. \& SILVA, J. P. (trads.). São Paulo, Livraria Santos Editora, 1990.

KIRKLIN, J. W. \& BARRAT-BOYES, B. G. - Aortic valve disease. In: KIRKLIN. J. W. Cardiac surgery. New York, Willey \& Sons, 1986. 376 p.

4 KRATZ, J. Avaliação e tratamento da doença valvar tricúspide. In: ARAÚJO, M. A. C. \& LANA, R. L. (trads.) Clínicas cardiológicas: doença cardiaca valvular. Rio de Janeiro, Interlivros, 1991. p. 427 437.

MACAMBIRA, R. P.; FRANCO, P. D. A.; THULER, L. C. S.; FRANCO, C. D. A. - Endocardites infecciosas. Cords, 2: 31-37, 1986. 
BRAILE, D. M.; VOLPE, M. A.; RAMIN, S. L.; SOUZA, D. R. S. - Tratamento cirúrgico das valvopatias. Parte 3. Rev. Bras. Cir. Cardiovasc., 9 (4): 181-192, 1994.

MASTERS, R. G.; PIPE, A. L.; BEDARD, J. P.; BRAIS, M. P.; GOLDSTEIN, W. F.; KOSHAL, A.; KEON, W. J. - Long-term clinical results with the lonescu-Shiley pericardial xenograft. J. Thorac. Cardiovasc. Surg., 101: 81-89, 1991.

27 MILLER, D. C. - Predictors of outcome in patients with prosthetic valve endocarditis (PVE) and potential advantage of homograft aortic root replacement for prosthetic ascending aortic valve-graft infections. J. Cardiac. Surg., 5: 53-62, 1990.

28 MILLS, S. A. - Surgical management of infective endocarditis. Ann. Surg., 195: 367-383, 1982.

29 NÚNEZ, L.; DE LA LLANA, R.; AGUADO, M. G.; IGLESIAS, A.; LARREA, J. L.; CALEMIN, D. Bioprosthetic valve endocarditis: indicators for surgical intervention. Ann. Thorac. Surg., 35: 262270, 1983.

30 O'BRIEN, M. F. - Implantation techniques and sizing of free sewn AVR and aortic root replacement. In: KLARK, D. R., (ed.) Transplantation techniques and use of cryopreserved allograft cardiac valves and vascular tissue. A Symposium and Laboratory for Surgeons, Atlanta, Sept, 1988.

31 PELLETIER, L. C.; CARRIER, M.; LECLERC, Y.; LEPAGE, G.; DEGUISE, P.; DYRDA, I. - Porcine versus pericardial bioprostheses: a comparison of

late results in 1,593 patients. Ann. Thorac. Surg., 47: 352-361, 1989.

32

REID, C. L. \& RAHIMTOOLA, S. H. - Endocardite infecciosa: papel da ecocardiografia, cateterização cardíaca e intervenção cirúrgica. Conceitos Modernos sobre Doenças Cardiovasculares, 5 (4), 1986.

REUL, G. L; COOLEY, D. A.; DUNCAN, J. M.; FRAZIER, O. H.; HALLMANN, G. L.; LIVESAY, J. J.; OTT, D. A.; WALKER, W. E. - Valve failure with lonescuShiley bovine pericardium bioprosthesis: analysis of 2680 patients. J. Vasc. Surg., 2: 192-204, 1985.

34 RICHARDSON, J. V.; KARP, R. B.; KIRKLIN, J. W.; DISMUKES, W. E. - Treatment of infective endocarditis: a 10-year comparative analysis. Circulation, 58: 589-597, 1978.

ROSS, D. - Allograft root replacement for prosthetic endocarditis. J. Cardiac. Surg., 5: 68-72, 1990.

WILSON, W. R.; DANIELSON, G. K.; GULIANI, E. R.; WASHINGTON, J. A.; JAUMIN, P. M.; GERACI, J. E. - Valve replacement in patients with active infective endocarditis. Circulation, 58: 585-588, 1978.

37 ZHU-XIADONG, GUO-JIAQING, CHEN-YING-CHUN, TANG-CHENG-JUNXUE-GANXING. - Ten-year experience with pericardial xenograft valves. $J$. Thorac. Cardiovasc. Surg., 95: 572-576, 1988. 